\title{
Routine upstream versus selective down stream use of tirofiban in non-ST elevation myocardial infarction patients scheduled for early invasive therapy; a randomized comparison
}

\author{
Saman Rasoul · Jan Paul Ottervanger · Menko Jan de Boer • \\ Jan Henk E. Dambrink · Harry Suryapranata · Jan C. A. Hoorntje • \\ A. T. Marcel Gosselink - Arnoud W. J. van ' $t$ Hof $\cdot$ on behalf of the ELISA study group
}

Published online: 2 March 2007

(C) Springer Science+Business Media, LLC 2007

\begin{abstract}
Background Despite their proven beneficial effects and inclusion in the guidelines, glycoprotein (GP) IIb/ IIIA blockers are underused in daily practice in patients with non ST-segment elevation acute coronary syndrome (NSTE ACS). This study combines the data from two randomized controlled trials, comparing routine upstream versus selective down stream use of tirofiban in patients with NSTE ACS.

Methods Inclusion criteria for both studies (ELISA-1 and 2) were angina pectoris, with ST depression $>1 \mathrm{~mm}$ and or a positive cardiac biomarkers. All patients were scheduled for coronary angiography. The primary and secondary end points for both studies were enzymatic infarct size (LDHQ48) and initial TIMI flow of the culprit lesion respectively.

Results From August 2000 to January 2005, 273 patients were randomized to routine upstream tirofiban and 275 patients to selective down stream use of tirofiban. Selective down stream tirofiban was used in 55 patients $(20 \%)$. Patients in the upstream group more often had a patent culprit lesion ( $65 \%$ vs. $50 \%$, $P=0.003)$ and a significantly smaller enzymatic infarct size, LDHQ48 median (25-75\%): 125 (55-309) vs. 189 (68-504) IU/l, $P=0.006$ as compared to the selective down stream group. Subgroup analysis showed that routine upstream tirofiban was particularly effective in
\end{abstract}

S. Rasoul · J. P. Ottervanger · M. J. de Boer

J. H. E. Dambrink · H. Suryapranata .

J. C. A. Hoorntje - A. T. M. Gosselink .

A. W. J. van 't Hof $(\varangle)$

Department of Cardiology, Isala Klinieken, Groot

Wezenland 20, 8011 JW Zwolle, The Netherlands

e-mail: v.r.c.derks@isala.nl males, patients with a positive troponin on admission and in those not pretreated with clopidogrel.

Conclusion Routine upstream GP IIb/IIIa is mainly effective in patients with elevated troponin on admission and those not pretreated with clopidogrel. Large scale randomized trials are needed to evaluate the effect of GP IIb/IIIa blockers on top of clopidogrel pretreatment on major adverse cardiac events.

Keywords Non ST-segment elevation ACS . Antithrombotic therapy · Glycoprotein IIb/IIIa inhibitor · Tirofiban

\section{Introduction}

Non-ST elevation acute coronary syndrome (NSTE ACS) are due to an acute or sub acute primary reduction of myocardial oxygen supply provoked by disruption of an atherosclerotic plaque associated with inflammation, thrombosis, vasoconstriction and microembolization. Treatment of this syndrome has been greatly improved since the introduction of anti-thrombotic agents and the aggressive use of cardiac catheterization in patients at high risk of adverse events. Both the European and American guidelines state that an initial invasive strategy and treatment with glycoprotein (GP) IIb/IIIa blockers are indicated in patients at high risk of adverse events [1,2]. This means that the majority of patients will be candidates for angiography.

A recent Meta analysis showed the beneficial effects GP IIb/IIIa inhibitor, particularly if intervention was performed during GP IIb/IIIa inhibitor infusion [3].

The current study describes enzymatic infarct size and angiographic outcome in high risk patients with 
NSTE ACS, randomized to routine upstream versus selective down stream use of tirofiban, all planned for angiography within $50 \mathrm{~h}$ of admission.

\section{Methods}

This study pooled the data from two separate randomized trials from our Institution: the Early or Late Intervention in unStable Angina- (ELISA) 1 and ELISA-2 trial. In- and exclusion criteria have been described and were the same for both studies [4, 5]. In brief, patients with symptoms of chest pain lasting more than $30 \mathrm{~min}$ in the previous $24 \mathrm{~h}$ before admission and either or both ST segment depression (1 $\mathrm{mm}$ or more) or a positive cardiac biomarkers (troponin $\mathrm{T}$ or $\mathrm{CKMB}$ ) were included. Patients in cardiogenic shock or contraindications for anti-platelet therapy or coronary angiography were excluded. Both trials randomized patients to routine upstream therapy with tirofiban or selective downstream therapy. The design of ELISA-1 and ELISA-2 differed only with regard to the timing of angiography and the upstream use of clopidogrel $[4,5]$. In the ELISA-1 study; patients with non-STEMI were randomized to either early angiography without tirofiban (loading dose of $10 \mu \mathrm{g} / \mathrm{kg}$ bolus followed by $0.15 \mu \mathrm{g} /$ $\mathrm{kg} / \mathrm{min}$ maintenance, for at least $12 \mathrm{~h}$ in case PCI was performed) pre-treatment (Early strategy) or to delayed angiography after $24-48 \mathrm{~h}$ pre-treatment with tirofiban (Late strategy). In the ELISA-2, non-STEMI patients were randomized to pre-treatment with dual (aspirin, clopidogrel $600 \mathrm{mg}$ ) or triple antiplatelet therapy (aspirin, clopidogrel $300 \mathrm{mg}$, and tirofiban loading dose of $10 \mu \mathrm{g} / \mathrm{kg}$ bolus followed by $0.15 \mu \mathrm{g} / \mathrm{kg} /$ min maintenance, for at least $12 \mathrm{~h}$ in case PCI), followed by angiography after $24-48 \mathrm{~h}$ later.

In both studies, the use of tirofiban downstream in the selective downstream group was left at the discretion of the operator. For both studies, enzymatic infarct size, defined as the cumulative release of LDH during the first $48 \mathrm{~h}$ after admission $\left(\mathrm{LDHQ}_{48}\right)$, was the primary end point and initial TIMI flow of the culprit coronary lesion, as defined by the TIMI criteria [6], was a pre specified secondary end point. Both endpoints were assessed by an independent core lab (Diagram, Zwolle, the Netherlands) by technicians blinded to randomization or clinical data. The analyses of both end point parameters have been described in details previously $[4,5]$.

Safety and outcome

Major bleeding was defined as the need for at least 2 units of blood and a fall in haemoglobin of more than
$2 \mathrm{mmol} / \mathrm{l}$, corrective groin surgery, gastro-intestinal bleeding, stroke or retroperitoneal bleeding. Clinical outcome was assessed at 30 day follow-up.

\section{Statistical analysis}

$\mathrm{LDHQ}_{48}$, the primary end point, was compared using the Mann-Whitney test and expressed as median and 25-75 percentiles. The initial TIMI flow of the culprit vessel was compared using Chi square analysis. The Fisher's exact test was used when the expected cell value was $<5$. Data were analysed according to the intention to treat principle. A $P$ value $<0.05$ was considered statistically significant. All tests were two-sided.

\section{Results}

From April 2000 to December 2001 and from September 2002 to January 2005, 548 consecutive patients were included in either the ELISA-1 (2000-2002) or ELISA-2 trial (2002-2005). A total of 273 patients were randomized to early initiation of tirofiban, upstream group, and 275 patients to selective down stream use tirofiban. No significant differences in baseline characteristics were present between the two groups (Table 1). Time to angiography was shorter in the selective downstream group, $16 \mathrm{~h}$ vs. $28 \mathrm{~h}$.

\section{Enzymatic infarct size}

Enzymatic infarct size as assessed by $\mathrm{LDHQ}_{48}$ is shown in Table 2 and Fig. 1. Enzymatic infarct size was not assessed in 65 patients (12\%) who had no coronary artery disease on angiography. In the remaining 483 patients, $\mathrm{LDHQ}_{48}$ could be assessed in 404 patients (84\%) and was 125 (55-309) median (25-75\%) in the upstream group as compared to $189(68-504)$ IU/1 in the selective down stream group $(P=0.006)$. Subgroup analysis, with regard to enzymatic infarct size, showed that tirofiban upstream use was especially effective in males, in patients with an elevated troponin on admission and those who did not receive upstream clopidogrel (Fig. 2).

\section{Angiographic parameters}

Coronary angiography was performed in $98 \%$ of the patients. Sixty-five patients $(12 \%)$ had no coronary artery disease. Multivessel disease was present in 55\% and $51 \%$ in the upstream and selective downstream 
Table 1 Baseline

MI, myocardial infarction; PCI, percutaneous coronary intervention; $\mathrm{CABG}$, coronary artery bypass grafting; Syst BP, systolic blood pressure

\begin{tabular}{lllr}
\hline Variables & Selective downstream $(n=275)$ & Upstream $(n=273)$ & $P$ value \\
\hline Age & $63.85 \pm 10.17$ & $62.96 \pm 11.11$ & 0.332 \\
Female gender & $76 / 275(28 \%)$ & $84 / 273(31 \%)$ & 0.420 \\
Diabetes mellitus & $49 / 274(18 \%)$ & $40 / 270(15 \%)$ & 0.333 \\
Hypertension & $130 / 266(4839 \%)$ & $113 / 261(43.3 \%)$ & 0.199 \\
Smoking & $104 / 255(40.8 \%)$ & $95 / 256(37.1 \%)$ & 0.394 \\
Hypercholesterolemia & $104 / 234(44.4 \%)$ & $95 / 222(42.8 \%)$ & 0.722 \\
Previous MI & $52 / 275(18.9 \%)$ & $50 / 273(18.3 \%)$ & 0.858 \\
Previous PCI & $46 / 275(16.7 \%)$ & $51 / 273(18.7 \%)$ & 0.549 \\
Admission angio (min 25-75\%) & $16.28(5.8-24.76)$ & $28.325(18.9-52.53)$ & $<0.001$ \\
Systolic BP & $148.84 \pm 25.46$ & $145.17 \pm 22.74$ & 0.077 \\
Troponin T $>0.05$ & $181 / 247(73.3 \%)$ & $153 / 235(65.1 \%)$ & 0.052 \\
Timi risk score & $2.74 \pm 1.44$ & $2.71 \pm 1.35$ & 0.827 \\
St depression $>1$ mm & $152 / 272(55.9 \%)$ & $159 / 272(58.5 \%)$ & 0.544 \\
Clopidogrel pre-treatment & $166 / 275(60 \%)$ & $162 / 273(59 \%)$ & 0.81 \\
\hline
\end{tabular}

Table 2 Enzyme release

\begin{tabular}{llll}
\hline Variables & $\begin{array}{l}\text { Selective } \\
\text { downstream } \\
(n=275)\end{array}$ & $\begin{array}{l}\text { Upstream } \\
(n=273)\end{array}$ & $P$ value \\
\hline Peak CK & $207(107-472)$ & $176(94-413)$ & 0.11 \\
Peak CK MB & $28(16-66)$ & $25(16-53)$ & 0.29 \\
LDHQ $_{48}$ & $189(68-503.75)$ & $125(55-309)$ & 0.006 \\
\hline
\end{tabular}

CK, creatine kinase; CK-MB, creatine kinase MB mass; $\mathrm{LDHQ}_{48}$, Enzymatic infarct size (area under the lactate dehydrogenase release over $48 \mathrm{~h}$ curve); Data are expressed as median and 25-75 percentiles

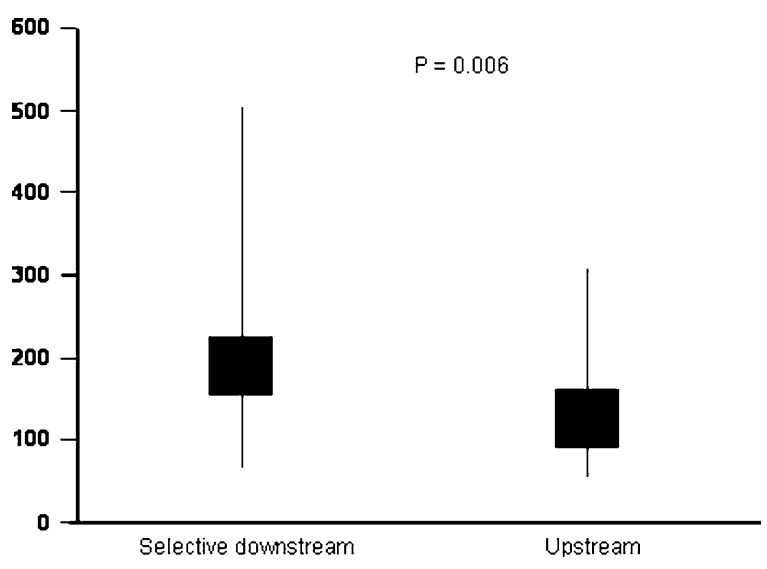

Fig. 1 Enzymatic infarct size

group respectively. Sixty-five percent of the patients in the upstream group had initial TIMI 3 flow of the culprit lesion compared to $50 \%$ in the selective downstream group $(P=0.003)$. Initial thrombus was present in $8 \%$ in the upstream group, as compared to $13 \%$ in the selective downstream group $(P=0.09)$.

Routine upstream tirofiban tended to result in a lower enzymatic infarct size in patients treated with medical management alone or who underwent CABG
(Fig. 3). However, the overall benefit of routine upstream tirofiban in initial TIMI 3 flow was seen in the patients who were treated with medical management (63.2\% vs. $35.7 \% ; P=0.045)$ alone or underwent coronary artery bypass grafting $(77.8 \%$ vs. $50.0 \%$; $P=0.025)$.

Selective downstream use of tirofiban

Selective downstream tirofiban was used in $55 / 275$ patients $(20 \%)$. In eight patients (3\%) upstream tirofiban was given because of persistent symptoms of ischemia before angiography. The other 47 patients $(17 \%)$ were treated with tirofiban after angiography or PCI (downstream).

\section{Medication}

On discharge the fast majority of the patients received Aspirin $(88 \%$ vs. $88 \% ; P=0.9)$, Beta blocker ( $86 \%$ vs. $85 \% ; P=0.8$ ), Statins $67 \%$ vs. $75 \% ; P=0.047)$ and Clopidogrel (57\% vs. $58 \% ; P=0.15)$ in the upstream and selective downstream group, respectively.

\section{Clinical outcome}

Thirty day follow-up was available in 544 (99\%) of patients. At 30 days follow-up, the incidence of death or recurrent myocardial infarction was present in $2.9 \%$ of patients in the upstream group versus $4.4 \%$ in the selective downstream group, $P=0.36$ (Table 3 ).

\section{Safety}

Major bleeding was not significantly different between the two groups and it was present in 33 patients $(12 \%)$ in the upstream group and 24 patients $(9 \%)$ in the selective downstream group (Table 3). CABG related 
Fig. 2 Enzymatic infarct size in different subgroup patients

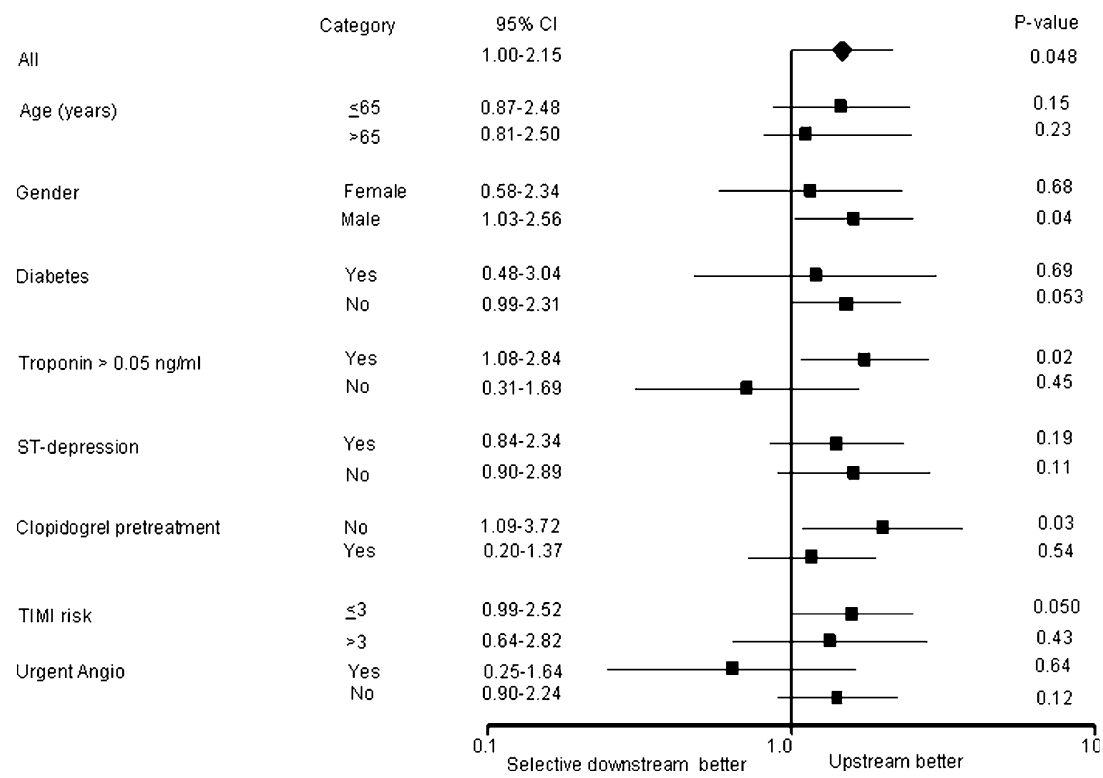

Table 3 Thirty day outcome

\begin{tabular}{llll}
\hline Events 30 days & $\begin{array}{l}\text { Selective } \\
\text { downstream } \\
(n=272)\end{array}$ & $\begin{array}{l}\text { Upstream } \\
(n=272)\end{array}$ & $P$ value \\
\hline Death & $3 / 272(1 \%)$ & $3 / 272(1 \%)$ & 1.000 \\
Reinfarction & $9 / 272(3 \%)$ & $6 / 272(2 \%)$ & 0.43 \\
Death or reinfarction & $12 / 272(4 \%)$ & $8 / 272(3 \%)$ & 0.36 \\
Bleeding & $24 / 272(9 \%)$ & $33 / 272(12 \%)$ & 0.21 \\
CABG related & $18 / 272(7 \%)$ & $24 / 272(9)$ & 0.34 \\
Surgical exploration & $3 / 272(1 \%)$ & $5 / 272(2 \%)$ & 0.51 \\
\hline
\end{tabular}

CABG, coronary artery bypass grafting

culprit lesion, as compared to selective downstream use only.

Limited data are available concerning the benefit of upstream use of GP IIb/IIIa blockers in NSTE ACS. Substudies of the PRISM-plus and the TACTICS TIMI 18 , studies showed that upstream therapy resulted in a better TIMI flow of the culprit coronary artery, a reduction of thrombus load and a better extent of myocardial perfusion before angiography, thereby showing the mechanism of benefit of upstream therapy $[7,8]$. The EVEREST trial compared the effect of upstream use of tirofiban versus downstream high-dose bolus tirofiban and abciximab in high risk NSTE ACS patients treated with PCI. Upstream tirofiban was associated with an improved tissue level perfusion and less troponin release after PCI [9].

Recently, two large randomized trials, JSAR-REACT 2 and ACUITY Timing trial, showed conflicting results with regard to the benefit of upstream use of GP IIb/IIIa inhibitor in NSTE ACS patients [10,11]. In the ISAR-REACT 2 trial high risk NSTE ACS patients 
were randomized to either upstream abciximab $(N=1012)$ or placebo $(N=1010)$. Short-term composite of death, myocardial infarction or urgent target vessel revascularization was significantly lower in the patients receiving abciximab [10]. In the ISAR-REACT 2 however, only patients undergoing PCI were included, while patients who were treated conservatively or underwent CABG were excluded.

In our study, routine upstream tirofiban tended toward a lower enzymatic infarct size in patients treated with medical management alone or who underwent CABG (Fig. 3). Larger randomized trials are warranted to investigate the additional use GP IIb/IIIa inhibitors according to treatment strategy. With regard to initial TIMI 3 flow, routine upstream tirofiban was effective in all treatment strategies. Results of the PRISM PLUS study confirm these data [12]. Furthermore, both the American and European guidelines for the treatment of patients with NSTE ACS support the management with GP IIb/IIIa blockers in high risk patients with NSTE ACS $[1,2]$.

The ACUITY Timing trial looked at upstream versus cath-lab administration of GP IIb/IIIa blockers and showed somewhat mixed results. While upstream use of IIb/IIIa blockers was associated with fewer ischemic events, there was no difference in net clinical outcome between the two strategies [11]. However these are unpublished and the results should be interpreted with caution.

Our results show that GP IIb/IIIa were particularly effective in patients with elevated troponin and in males. This is consistent with a previous meta analysis and with the ISAR-REACT 2 trial [7, 10]. Furthermore, a cost-effective analysis showed the superiority of upstream use as compared to selective use of GP IIb/IIIa blockers in patients with moderate to high risk ACS patients [13]. The reason why upstream use is more effective than selective downstream use GP IIb/ IIIa blocker may not only be due to the prevention of thrombus formation. Goto et al. showed that GP IIb/ IIIa blockers are able to dissolve platelet aggregates, which already formed in high-shear milieu [14].

The use of GP IIb/IIIa inhibitors are mainly recommended in patients who undergo PCI, however, the current evidence does not support the use of GP IIb/ IIIa inhibition only in conjunction with PCI [15]. In addition, it is impossible to know at hospital admission which patients will later undergo PCI and thus physician should consider the use of GP IIb/IIIa blockers in all, at least high-risk, patients presenting with an NSTE ACS. Furthermore, the currently ongoing Early ACS study [16] with a comparable design as our study, has planned to include more than 10,000 patients to find a difference in a combined clinical endpoint. This trial will provide important evidence regarding the benefit of initiating GP IIb/IIIa inhibitor early after presentation with high-risk ACS.

Taken together, the results of our study and almost all the above mentioned trials support the routine upstream use of GP IIb/IIIa blockers in moderate to high risk patients with NSTE ACS. In addition, the use of these agents are recommended by both the American and the European guidelines.

\section{Limitations}

Not all the patients were pre-treated with clopidogrel, however according to the current guidelines pretreatment with clopidogrel is well recommended in patients with NSTE ACS. Another limitation of the study is that $\mathrm{LDHQ}_{48}$ is not an established end point in NSTE ACS trials. However, previous trials showed that enzymatic infarct size is well correlated with clinical parameters [17, 18]. Another limitation was that enzymatic infarct size was not available in $16 \%$ of the patients. Furthermore, LDH concentrations may be influenced by haemolysis and this may affect the real enzymatic infarct size. Although, 42 out of 57 patients had a CABG related bleeding, CABG was performed in most of the patients beyond $48 \mathrm{~h}$ after admission.

\section{Conclusion}

Routine early upstream use of the GP IIb/IIIa inhibitor, tirofiban, reduces enzymatic infarct size and is associated with a better initial patency of the culprit lesion in patients with NSTE ACS. This effect was most evident in males, those with elevated troponin on admission and those not pre-treated with clopidogrel. Large scale randomized trials are needed to evaluate the effect of GP IIb/IIIa blockers on top of clopidogrel pretreatment on major adverse cardiac events.

\section{References}

1. Silber S, Albertsson P, Aviles FF, Task Force for Percutaneous Coronary Interventions of the European Society of Cardiology et al (2005) Guidelines for percutaneous coronary interventions. The Task Force for Percutaneous Coronary Interventions of the European Society of Cardiology. Eur Heart J 26:804-847

2. Braunwald E, Antman E, Beasley J et al (2000) ACC/AHA guidelines for the management of patients with unstable angina and non-ST-segment elevation myocardial infarction: 
a report of the American College of Cardiology/American Heart Association Task Force on Practice Guidelines (Committee on the Management of Patients with Unstable Angina). J Am Coll Cardiol 36:970-972

3. Roffi M, Chew DP, Mukherjee D et al (2002) Platelet glycoprotein IIb/IIIa inhibition in acute coronary syndromes: gradient of benefit related to the revascularization strategy. Eur Heart J 23:1441-1448

4. van 't Hof AWJ, de Vries ST, Dambrink JHE et al (2003) A comparison of two invasive strategies in patients with non-ST elevation acute coronary syndromes: results of the Early or Late Intervention in unStable Angina (ELISA) pilot study. IIb/IIIa upstream therapy and acute coronary syndromes. Eur Heart J 24:1401-1405

5. Rasoul S, Ottervanger JP, de Boer MJ et al (2006) A comparison of dual versus triple antiplatelet therapy in patients with non ST segment elevation acute coronary syndromes. Results of the ELISA-2 trial. Eur Heart J 27:1401-1407

6. Chesebro JH, Knatterud G, Roberts R et al (1987) Thrombolysis In Myocardial Infarction (TIMI) trial, phase 1: a comparison between intravenous tissue plasminogen activator and intravenous streptokinase. Circulation 76:723-725

7. Zhao X-Q, Theroux P, Snapinn SM, Sax FL (1999) Intracoronary thrombus and platelet glycoprotein IIb/IIIa receptor blockade with Tirofiban in unstable angina or non-Qwave myocardial infarction. Angiographic results from the PRISM-PLUS trial (Platelet receptor inhibition for ischemic syndrome management in patients limited by unstable signs and symptoms). PRISM-PLUS Investigators. Circulation 100:1609-1615

8. Gibson CM, Singh KP, Murphy SA, TIMI Study Group et al (2004) Association between duration of tirofiban therapy before percutaneous intervention and tissue level perfusion (a TACTICS-TIMI 18 substudy). Am J Cardiol 94:492-494

9. Bolognese L, Falsini G, Liistro F et al (2006) Randomized comparison of upstream tirofiban versus downstream high bolus dose tirofiban or abciximab on tissue-level perfusion and troponin release in high-risk acute coronary syndromes treated with percutaneous coronary interventions: the EVEREST trial. J Am Coll Cardiol 47:522-528

10. Kastrati A, Mehilli J, Neumann FJ et al (2006) Abciximab in patients with acute coronary syndromes undergoing percutaneous coronary intervention after clopidogrel pretreatment: the ISAR-REACT 2 randomized trial. JAMA 295:1531-1538

11. Stone G (2006) ACUITY TIMING, presented at American college of Cardiology. Available at: http://acc06online.acc.org/Lectures.aspx? sessionId=67\&date $=13$

12. The Platelet Receptor Inhibition in Ischemic Syndrome Management in Patients Limited by Unstable Signs and Symptoms (PRISM-PLUS) Study Investigators: Inhibition of the platelet glycoprotein IIb/IIIa receptor with tirofiban in unstable angina and non-Q-wave myocardial infarction. N Engl J Med (1998) 338:1488-1497

13. Glaser R, Glick HA, Herrmann HC, Kimmel SE (2006) The role of risk stratification in the decision to provide upstream versus selective glycoprotein IIb/IIIa inhibitors for acute coronary syndromes: a cost-effectiveness analysis. J Am Coll Cardiol 47:529-537

14. Goto S, Tamura N, Ishida H (2004) Ability of anti-glycoprotein IIb/IIIa agents to dissolve platelet thrombi formed on a collagen surface under blood flow conditions. J Am Coll Cardiol 44:316-323

15. Pieper KS, Tsiatis AA, Davidian M et al (2004) Differential treatment benefit of platelet glycoprotein IIb/IIIa inhibition with percutaneous coronary intervention versus medical therapy for acute coronary syndromes: exploration of methods. Circulation 109:641-646

16. Giugliano RP, Newby LK, Harrington RA, EARLY ACS Steering Committee et al (2005) The early glycoprotein IIb/ IIIa inhibition in non-ST-segment elevation acute coronary syndrome (EARLY ACS) trial: a randomized placebo-controlled trial evaluating the clinical benefits of early frontloaded eptifibatide in the treatment of patients with non-STsegment elevation acute coronary syndrome-study design and rationale. Am Heart J 149:994-1002

17. Januzzi JL, Chae CU, Sabatine MS, Jang IK (2001) Elevation in serum troponin I predicts the benefit of tirofiban. J Thromb Thrombolysis 11:211-215

18. Roe MT, Christenson RH, Ohman EM, EARLY Investigators; Emergency Medicine Cardiac Research and Education Group et al (2003) A randomized, placebo-controlled trial of early eptifibatide for non-ST-segment elevation acute coronary syndromes. Am Heart J 146:993-998 